\title{
UM MODELO DE GESTÃO DE ATIVO/PASSIVO: APLICAÇÃO PARA FUNDOS DE BENEFÍCIO DEFINIDO COM ATIVOS DE FLUXO INCERTO
}

\author{
ASSET/LIABILITY MANAGEMENT MODEL: APPLICATION TO DEFINED \\ BENEFIT PENSION FUNDS WITH UNCERTAIN ASSET FLOWS
}

NICOLAS SOUDKI SAAD

Gestor de Fundos do HSBC Investments

E-mail: nicolas.s.saad@hsbc.com.br

\author{
CELMA DE OLIVEIRA RIBEIRO \\ Professora Doutora do Departamento de Engenharia \\ de Produção da Escola Politécnica \\ da Universidade de São Paulo - Campus Capital \\ E-mail: celma@usp.br
}

\section{RESUMO}

O artigo apresenta uma aplicaçāo de um modelo do tipo Gestão Ativo/Passivo (Asset/Liability Management) em um fundo de pensão brasileiro do tipo Benefício Definido. O modelo proposto consiste em uma ferramenta de gestão de carteiras que adota uma abordagem estática, em que a incerteza dos ativos é inserida no modelo na forma de uma penalidade que depende de um parâmetro de aversão ao risco e da volatilidade dos fatores de risco. Uma aplicação do modelo a um problema real de alocação de ativos financeiros indica que a ferramenta consiste em uma abordagem simples e eficiente para gestão de fundos de pensão.

Palavras-chave: Gestão Ativo/Passivo, Carteiras de Ativos, Finanças.

\section{ABSTRACT}

This paper presents an application of a new Asset/Liability Management model in a Brazilian Defined Benefit pension fund. The proposed model is a portfolio management tool that adopts a static approach. The uncertainty of assets is included in the model as a penalty that depends on a risk aversion parameter and on the volatility of risk factors. The application of the model to a real asset allocation problem shows that the tool represents a simple and accurate approach to pension fund management.

Keywords: Asset/Liability Management, Portfolio, Finance. 


\section{INTRODUÇÃO}

A metodologia de gestão de recursos de fundos de pensão tem evoluído significativamente no Brasil, e, a cada ano, torna-se maior a preocupaçāo em se fazer uma administração de recursos adequada às necessidades específicas de cada entidade de previdência complementar.

Dentre os fatores responsáveis por esse fenômeno destacam-se, em primeiro lugar, as quedas das taxas de inflação ocorridas após 1994. que permitiram que as projeções dos passivos dos planos de aposentadoria ganhassem confiabilidade. Além disso, as significativas quedas das taxas de juros reais' ocorridas após a flutuação cambial em 1999 provocaram uma reavaliação do paradigma de se fazer uma gestão de ativos voltada à superação de uma meta de rentabilidade atuarial do tipo IGP-M mais 6\%a.a., sem se considerar as particularidades do passivo de uma entidade. Por fim, a legislação aplicável às entidades fechadas de previdência complementar tem não somente enfatizado, mas exigido que a gestão de recursos dessas entidades seja cada vez mais adaptada às suas necessidades específicas.

Nesse contexto, este artigo apresenta uma aplicação de um modelo de gestão ativo/passivo que, através das projeçōes atuariais desenvolvidas para um plano de aposentadoria, considera as características específicas desse plano para propor uma carteira de ativos ideal. O modelo aqui apresentado se aplica a planos do tipo benefício definido, ou seja, planos que "garantem" o pagamento de um complemento de aposentadoria baseado em regras específicas e, que, portanto, possuem um passivo relacionado a esses compromissos.

Nos últimos anos tem havido uma importante migração dos planos de aposentadoria complementar oferecidos pelas empresas na direção da estrutura de contribuição definida, na qual o benefício do participante dependerá de sua poupança individual acumulada até a data da aposentadoria. Nessa estrutura, o plano não se compromete com o pagamento do benefício, mas com a gestão adequada dos recursos do participante e, portanto, não há um passivo financeiro. Apesar disso, ainda há muitos planos de benefício definido no Brasil, especialmente os mais antigos e de maior patrimônio. Além disso, algumas estruturas de planos de contribuição definida garantem um benefício após a aposentadoria, de modo que o modelo mantém sua aplicabilidade.

Na seção 2. contextualiza-se metodologicamente o artigo. Na parte 3, é apresentado o modelo originalmente proposto na literatura, bem como a adaptação desenvolvida. Após isso, apresenta-se sua implementação em um plano de aposentadoria e, finalmente, na seção 5 os resultados são analisados. A conclusão é apresentada na seção 6 .

\section{METODOLOGIA}

O objetivo deste trabalho é o de propor um modelo de otimização da carteira de ativos de um fundo de pensão baseado na literatura de finanças mas adequado à realidade da disponibilidade de ativos do mercado brasileiro. Adicionalmente. busca-se analisar os resultados da aplicação desse modelo numa situação real.

Dessa forma, o artigo caracteriza-se como pesquisa exploratória, à medida que tem por objetivo trazer uma maior compreensão do problema, propondo e analisando os resultados de uma metodologia de solução.

O trabalho divide-se em três partes considerando-se o ponto de vista metodológico. Na primeira etapa, elabora-se uma pesquisa bibliográfica dos modelos financeiros de otimização aplicáveis à situação em questão. A seguir, propōe-se uma metodologia para adaptar os modelos encontrados na literatura à disponibilidade de alternativas de investimento no mercado brasileiro. Essa metodologia constitui uma generalização da modelagem para a situação em que há diversos instrumentos de mercado disponiveis e, embora tenha sido desenvolvida visando à aplicação ao mercado brasileiro, ela é válida para qualquer situação em que haja alternativas de ativos cuja variação dependa de diferentes indexadores. A parte final do trabalho constitui uma análise dos resultados aplicados a um fundo de pensão real.

A contribuição do presente trabalho, portanto, é o desenvolvimento da pesquisa para a adequada gestāo financeira de longo prazo de recursos de fundos de pensão ou, mais genericamente. de recursos que estejam atrelados ao cumprimento de obrigações futuras. As limitações do trabalho estão ligadas às hipóteses simplificadoras que permitem o desenvolvimento e aplicação da metodologia proposta, dentre as quais se podem citar a utilização de modelos estáticos de otimização e a não consideração das dinâmicas de difusão das variáveis financeiras envolvidas.

1 Especialmente quando medida utilizando-se o CDI comparado com o IGP-M 


\section{MODELO DE DEDICAÇÃO ADAPTADO}

O uso de modelos matemáticos para problemas de gestão de ativo/passivo (Asset/Liability Management) não é recente. Há uma extensa literatura na área de finanças cujo objetivo é encontrar uma carteira de investimento ótima, segundo alguma medida de desempenho, o que recai em modelos de otimizaçāo (JUDICE et al., 2003). (KONNO e YAMAZAKI, 1991). (STEINBACK, 2001), (MARKOWITZ, 1952). Uma peculiaridade dos modelos aplicáveis a esse tipo de problema é que as decisões devem contemplar um horizonte de tempo longo, o que pode levar a modelos de grande porte de difícil resolução quando se considera a incerteza dos ativos analisados.

Construídos com a finalidade de determinar carteiras de máxima rentabilidade cujos ativos possuam a mesma sensibilidade às taxas de juros que o passivo, alguns desses modelos - denominados modelos de imunizaçăo - não permitem a inclusão na carteira de ativos com fluxo incerto (DAHL, MEERAUS e ZENIOS, 1993). (SAAD e RIBEIRO, 2004). Para tratar do risco de descasamento entre ativos e passivos de planos Benefício Definido pode-se empregar o modelo de dedicação. Conforme encontrado na literatura (DAHL, MEERAUS e ZENIOS, 1993), esse modelo é derivado do modelo denominado casamento de fluxos de caixa ("cash flow matching"). que consiste em construir uma carteira de ativos de renda fixa cujos fluxos de caixa se igualem, exatamente, aos pagamentos projetados para o passivo. Quando definido dessa forma, o problema não deve apresentar soluções viáveis, especialmente quando abordado em um universo relativamente limitado de ativos como o mercado brasileiro de renda fixa. Dessa forma, a restrição de casamento perfeito entre fluxos de ativo e passivo deve ser relaxada de modo a permitir a solução do problema, relaxamento esse que leva à formulação do modelo de Dedicação (Dedication). Conseqüentemente, o modelo de dedicação consistiria em se gerar a carteira de menor preço de mercado cujos fluxos de caixa precedam os desembolsos projetados para o passivo. Seja a notação:

$i$ : índice dos ativos;

$t:$ índice das datas nas quais há fluxos de caixa de ativos:

$\tau$ : índice das datas nas quais há fluxos de caixa de passivos:

$x_{i}$ : quantidade de ativo i na carteira (váriaveis de decisão):

$P_{i}$ : preço de mercado do ativo $t i$;

$\Delta \tau$ : intervalo de tempo entre as datas $\tau$ e $\tau-1$ :

$L_{\tau}$ : desembolso projetado para o passivo na data $\tau$;

$s_{\tau}$ : superávit (excesso de caixa) na data $\tau$;

$\rho:$ taxa de reinvestimento, que varia ao longo do tempo:

$C_{i, j}$ : fluxo de caixa do ativo i na data $\mathrm{t}$;

$D_{i, x}$ : fluxo de caixa do ativo i na data $t$, reinvestido na data t $\mathrm{e} \tau$.

Para a data $\tau, D_{i . t}$ pode ser calculado aplicando-se a taxa de reinvestimento $(\rho)$ sobre cada fluxo de caixa do ativo $i$ que ocorra entre as datas t e $\tau$ :

$$
D_{i t}=\sum_{r \in[r-1, x]} C_{i t} \times\left(1+\rho^{x-1}\right)
$$

O superávit para uma data específica $\tau$ é, então, definido como a soma dos fluxos de caixa que ocorrem entre $\tau$-1 e $\tau$ reinvestidos mais o superávit de $\tau-1$ reinvestido menos o desembolso do passivo que ocorre na data $\tau$ :

$$
s_{\tau}=\sum_{i} D_{i \tau} x_{i}+s_{\tau-1} \times(1+\rho)^{\Delta t}-\mathrm{L}_{\tau}
$$

Para concluir a estrutura do modelo, é necessário impor a nāo negatividade de cada superávit s conforme definido na equação (2). pois isso garante a condição básica do modelo de que os fluxos de caixa dos ativos precedam os desembolsos do passivo. Dessa forma, o modelo é o seguinte:

\section{Modelo de Dedicação}

$$
\begin{aligned}
& \min \sum_{i} P_{i} x_{i}+s_{0} \\
& \text { s.a.: } \\
& \sum_{i} D_{i \tau} x_{i}+s_{\tau-1} \times(1+\rho)^{\Delta t}=\mathrm{L}_{\tau}+s_{\tau}, \forall \tau \\
& s_{\tau} \geq 0, \forall \tau \\
& x_{i} \geq 0, \forall i
\end{aligned}
$$


Como o modelo é linear nas variáveis de decisão, é possível utilizar técnicas de programação linear para sua resolução. O número de restriçōes de igualdade do modelo é igual ao número de períodos considerado - o horizonte de planejamento $\mathrm{H}$ - e o número de variáveis é $(\mathrm{N}+\mathrm{H})$, em que $\mathrm{N}$ é o número de ativos considerados, ou seja, as dimensões do modelo dependem, exclusivamente, do número de variáveis e do horizonte de planejamento, simplificando, significativamente, sua resolução. É interessante notar, nesse ponto, que o modelo de dedicação responde, adequadamente, a um dos problemas fundamentais da gestão de ativos em mercados com liquidez limitada. Isso ocorre porque, sob esse modelo, não há necessidade de rebalanceamento contínuo da carteira para que as restrições continuem a ser atendidas ao longo do tempo, mesmo que os preços dos ativos e prazos dos fluxos de caixa se modifiquem. Portanto, não há a obrigatoriedade de se transacionar ativos para que as restriçōes continuem a ser satisfeitas, mesmo que a solução ótima se modifique. Colocando de outra forma, o modelo ativamente prefere o risco de reinvestimento ao risco de mercado, o que é desejável quando se trabalha em um ambiente com freqüentes e relevantes flutuações de liquidez.

$\mathrm{Na}$ forma apresentada, o modelo possui duas importantes limitaçōes. Em primeiro lugar, projeçōes econômicas de longo prazo são necessárias para determinar nāo apenas os fluxos de caixa esperados para ativos e passivos, mas também as taxas de reinvestimento. Tais projeções incluem, especialmente, as taxas de inflação e taxas de juros. O problema disso advindo é que há, naturalmente, muita incerteza em relação às variáveis projetadas para prazos mais longos, ao mesmo tempo em que o modelo não permite tais imprecisões. Em segundo lugar, após se introduzir em (3) os fluxos de caixa projetados, o modelo não fará distinção entre os diferentes ativos que geram os fluxos de caixa. Portanto, o modelo não considera os riscos de investimento de cada instrumento em relação ao passivo.

A alternativa usual aos modelos determinísticos como o apresentado, e que tem tido destaque na literatura recente, são os modelos de otimização estocástica que pressupõem que os fluxos de caixa de ativos e passivos evoluem segundo processos estocásticos conhecidos, descritos através de cenários discretos (PFLUG, 2001) (BOULIER et al., 2001). (CAIRNS e PARKER, 1997). Nesse caso, as decisōes, em cada período, dependem do estado em que se encontram os fluxos de caixa naquele instante e com isso o modelo passa a ter grandes dimensões, impondo o emprego de técnicas sofisticadas para implementação e uso em problemas reais (PAPI e SBARAGLIA, 2005). (FRANCOS et al., 2004). (BOENDER, 1997). A idéia central do modelo aqui proposto é incorporar o conceito de risco ao processo de otimização, sem recair em problemas de grande complexidade computacional.

A seguir, apresenta-se uma adaptação do modelo de dedicação que permite lidar com risco através de dois parâmetros: a aversão ao risco do investidor e a volatilidade dos ativos. A idéia é introduzir uma penalidade que será subtraída de cada fluxo de caixa nominal esperado, ou seja, ao invés de considerar os fluxos de caixa originais em cada período, corrigem-se seus valores ao longo do tempo de acordo com a volatilidade do ativo e do parâmetro de aversão ao risco. A correção é apresentada nas equações (4) e (5). Note que a correção, também, se aplica à taxa de reinvestimento, o que, do ponto de vista prático, gera um interessante efeito de diversificação temporal dos vencimentos da carteira otimizada.

$\mathrm{CF}_{0}$ : fluxo de caixa esperado:

$\mathrm{CF}$ : fluxo de caixa corrigido;

$\lambda$ : parâmetro de aversão ao risco;

$T$ : período de tempo até o fluxo de caixa;

$\sigma$ : parâmetro de risco para o instrumento gerador do fluxo de caixa;

$\rho_{A}(g)$ : taxa de reinvestimento acumulada esperada em uma data g:

$\rho_{\mathrm{Eg} \rightarrow \rightarrow \mathrm{g}}:$ taxa de reinvestimento corrigida entre as datas $\mathrm{g}-1 \mathrm{e} \mathrm{g}$;

$\Delta G$ : intervalo de tempo entre $\mathrm{g}-1 \mathrm{eg}$.

$$
\begin{gathered}
C F=C F_{0} \times e^{-\lambda \times \mathrm{T} \sigma} \\
\rho_{E, g-1 \rightarrow g}=\frac{\rho_{A}(g)}{\rho_{A}(g-1)} \times e^{-\lambda \times \Delta g \times \sigma}
\end{gathered}
$$

Um aspecto importante a se destacar é que, apesar da não linearidade da correção proposta, o modelo final preserva a linearidade nas variáveis de decisão após a fixação dos parâmetros, e mantém o número de restrições e de variáveis. Por fim. cabe observar que, ao aplicar o ajuste proposto, a solução do modelo passa a ser parametrizada segundo o parâmetro de aversão ao risco $\lambda$, ou seja. diferentes carteiras ótimas são obtidas conforme varia o parâmetro de aversão ao risco. Há, é claro, a necessidade de se fixar, adequadamente, o valor desse parâmetro, o que tem sido feito, na prática, através do ajuste empírico baseado nos resultados, mais especificamente, comparando-se o valor da função objetivo otimizada com o valor total de ativos do plano, como será visto na aplicação que se segue. A definição dos parâmetros $\sigma$, por outro lado, pode ser mais quantitativa, uma vez que, na prática, trata-se de uma penalização aplicada à taxa de rentabilidade projetada para cada ativo. 


\section{UMA APLICAC̣ÃO A UM FUNDO DE PENSÃO BRASILEIRO}

\subsection{Breve Descrição}

O fundo de pensão para o qual foi aplicado o modelo consiste em um plano benefício definido criado em meados dos anos 60. O fundo era patrocinado por uma importante instituição financeira nacional, subseqüentemente adquirida por um grande banco internacional, e que optou por fechar o plano a novos entrantes e migrar para previdência aberta. Atualmente, portanto, não há entrada de novos participantes, tratando-se então de uma massa fechada, o que contribui para a estabilidade das projeções atuariais, característica bastante desejável quando se usa um modelo estático como o apresentado.

Uma outra característica importante do plano é que, após a aposentadoria, o benefício dos participantes é ajustado anualmente pela taxa de inflação medida pelo IGP-M. Esse fator de risco é fundamental para se definirem os índices de correção a serem aplicados para ajustar os fluxos de caixa conforme as equações (4) e (5) apresentadas anteriormente.

Atualmente, os resultados do modelo, com alguns ajustes às restriçōes, foram, efetivamente, implementados na carteira de ativos do plano e o modelo tem sido atualizado, para esse fim, em base mensal. Por questões de sigilo, algumas informaçōes aqui apresentadas são múltiplos das variáveis reais, o que não modifica a aplicabilidade do estudo nem as conclusões.

\subsection{Coleta de Dados}

A estimativa do passivo do plano foi elaborada pela área atuarial da empresa patrocinadora. A estimativa consiste nos fluxos de caixa anuais esperados para o plano, em moeda constante. As hipóteses atuariais e especificidades metodológicas utilizadas na projeção do passivo, no entanto, estão fora do escopo deste artigo. O prazo de projeção do passivo é o prazo esperado até a extinção do plano baseada na tabela atuarial de expectativa de vida, que é o ano de 2050, mas os fluxos de caixa nominais esperados deixam de crescer por volta do ano de 2013. O Gráfico 10 mostra os fluxos de caixa nominais mensais, que foram calculados com base nas estimativas anuais e nas projeçōes de inflação (apresentadas adiante). Os picos que podem ser observados no gráfico correspondem aos meses de dezembro e nada mais são do que a projeção de pagamento do $13^{\circ}$ salário que ocorre nesse mês.

O outro dado necessário para a otimização é o cenário econômico de longo prazo, e, mais especificamente, os valores esperados para os indexadores relevantes de ativos e passivos. Pelo lado do passivo, a única variável relevante é a taxa de inflação medida pelo IGP-M. Para os ativos, uma das variáveis mais relevantes é a taxa básica de juros (taxa Selic), que é necessária tanto para a projeção dos fluxos de caixa dos títulos pós-fixados indexados a essa taxa - as Letras Financeiras do Tesouro, ou LFTs, quanto para a projeção da taxa de reinvestimento ao longo do tempo. As outras variáveis necessárias são os índices de inflaçāo aos quais sāo indexados os títulos públicos, ou seja. IGP-M e IPCA. Os títulos indexados à inflação sāo as notas do Tesouro Nacional séries B e C (NTN-Bs e NTN-Cs)².

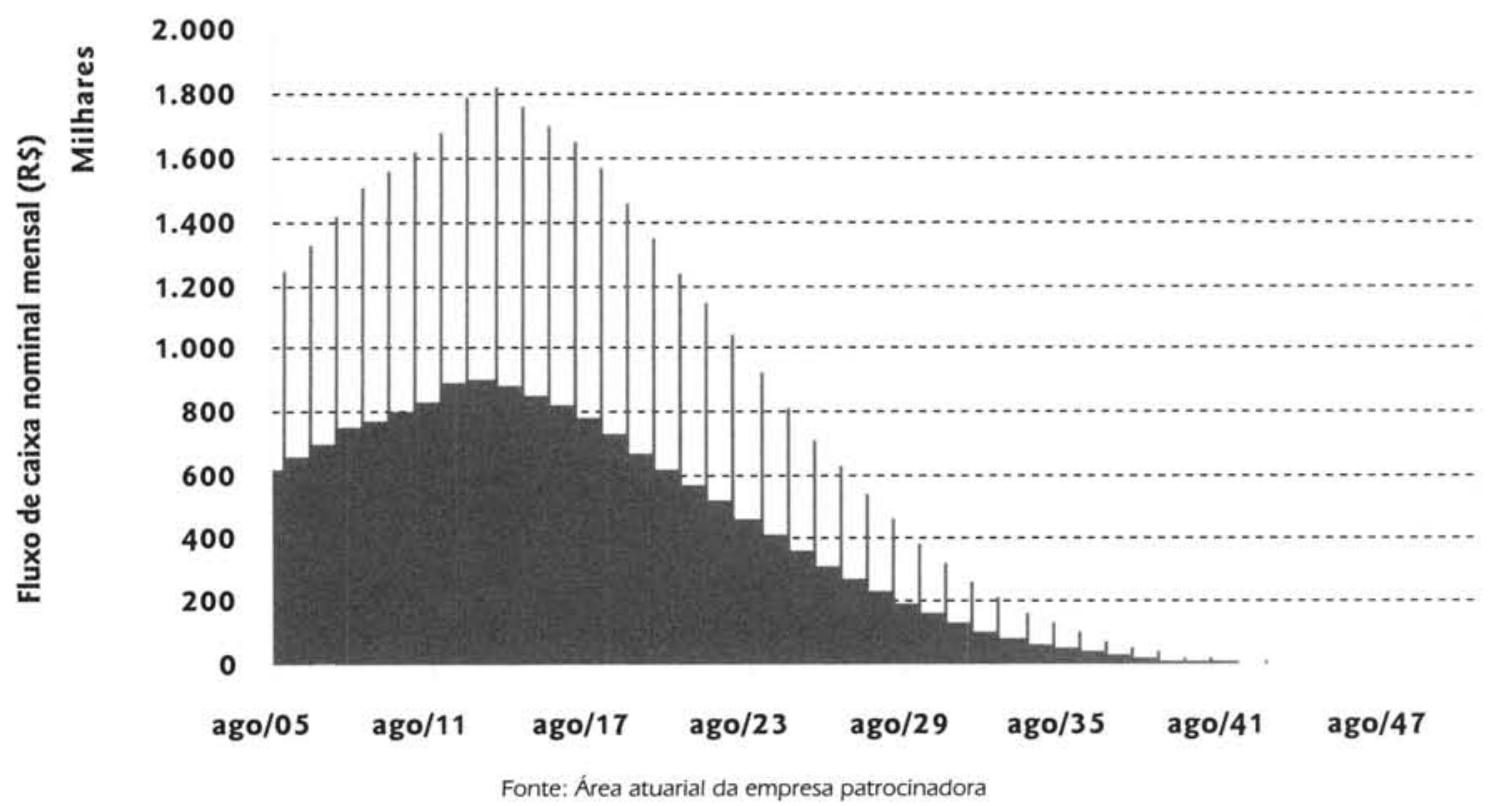

Gráfico 1

Fluxos de caixa nominais esperados

2 As NTN-Bs são indexadas à variação do IPCA, enquanto as NTN-Cs sâo indexadas à variação do IGP-M. 
A área econômica da empresa de gestão de ativos do Banco elaborou a projeção de longo prazo para essas variáveis até o ano de 2050, baseada em seu cenário básico de longo prazo para a economia brasileira. A Tabela 10 resume os dados do cenário. Conforme foi mencionado, há, intrinsecamente, um grande grau de incerteza em relação a essas projeçōes, que são, adequadamente, tratadas pelo modelo de dedicação ajustado.

Os ativos utilizados na otimização foram aqueles para os quais a ANDIMA - Associação Nacional das Instituições de Mercado Aberto publica informaçōes diárias de preços. A Tabela 20 e os Gráficos 20 e 30 resumem as emissões disponíveis na data da otimização (10/agosto/2005). Para a otimização aqui apresentada, utilizaram-se, apenas, os ativos indexados à taxa Selic, os ativos indexados às taxas de inflação mencionadas e os ativos pré-fixados ${ }^{3}$.

Tabela 1 || Projeções Econômicas de Longo Prazo

\begin{tabular}{|c|c|c|c|c|c|c|c|}
\hline Ano & IPCA & IGP-M & Selic & Ano & IPCA & IGP-M & Selic \\
\hline 2005 & $5,0 \%$ & $2,0 \%$ & $19,0 \%$ & 2028 & $2,7 \%$ & $2,6 \%$ & $6,8 \%$ \\
\hline 2006 & $4,9 \%$ & $5,5 \%$ & $16,5 \%$ & 2029 & $2,4 \%$ & $2,4 \%$ & $6,4 \%$ \\
\hline 2007 & $4,1 \%$ & $5,4 \%$ & $15,1 \%$ & 2030 & $2,3 \%$ & $2,4 \%$ & $5,4 \%$ \\
\hline 2008 & $4,0 \%$ & $5,2 \%$ & $14,5 \%$ & 2031 & $2,5 \%$ & $2,6 \%$ & $6,6 \%$ \\
\hline 2009 & $3,9 \%$ & $5,0 \%$ & $13,9 \%$ & 2032 & $2,5 \%$ & $2,6 \%$ & $7,6 \%$ \\
\hline 2010 & $3,8 \%$ & $4,8 \%$ & $13,4 \%$ & 2033 & $2,2 \%$ & $2,2 \%$ & $6,2 \%$ \\
\hline 2011 & $4,1 \%$ & $5,1 \%$ & $14,2 \%$ & 2034 & $2,2 \%$ & $2,2 \%$ & $5,2 \%$ \\
\hline 2012 & $4,0 \%$ & $4,9 \%$ & $14,7 \%$ & 2035 & $2,3 \%$ & $2,4 \%$ & $5,4 \%$ \\
\hline 2013 & $3,3 \%$ & $4,0 \%$ & $12,5 \%$ & 2036 & $2,1 \%$ & $2,2 \%$ & $4,2 \%$ \\
\hline 2014 & $3,2 \%$ & $3,8 \%$ & $11,1 \%$ & 2037 & $2,3 \%$ & $2,4 \%$ & $4,4 \%$ \\
\hline 2015 & $3,4 \%$ & $4,0 \%$ & $11,0 \%$ & 2038 & $2,5 \%$ & $2,6 \%$ & $5,6 \%$ \\
\hline 2016 & $3,0 \%$ & $3,5 \%$ & $9,2 \%$ & 2039 & $2,3 \%$ & $2,4 \%$ & $5,4 \%$ \\
\hline 2017 & $3,3 \%$ & $3,7 \%$ & $9,1 \%$ & 2040 & $2,3 \%$ & $2,4 \%$ & $5,4 \%$ \\
\hline 2018 & $3,5 \%$ & $3,9 \%$ & $10,1 \%$ & 2041 & $2,5 \%$ & $2,6 \%$ & $6,6 \%$ \\
\hline 2019 & $3,1 \%$ & $3,5 \%$ & $9,4 \%$ & 2042 & $2,5 \%$ & $2,6 \%$ & $7,6 \%$ \\
\hline 2020 & $3,0 \%$ & $3,3 \%$ & $9,0 \%$ & 2043 & $2,2 \%$ & $2,2 \%$ & $6,2 \%$ \\
\hline 2021 & $3,2 \%$ & $3,5 \%$ & $9,9 \%$ & 2044 & $2,2 \%$ & $2,2 \%$ & $5,2 \%$ \\
\hline 2022 & $3,1 \%$ & $3,3 \%$ & $10,6 \%$ & 2045 & $2,3 \%$ & $2,4 \%$ & $5,4 \%$ \\
\hline 2023 & $2,6 \%$ & $2,7 \%$ & $8,8 \%$ & 2046 & $2,1 \%$ & $2,2 \%$ & $4,2 \%$ \\
\hline 2024 & $2,5 \%$ & $2,6 \%$ & $7,5 \%$ & 2047 & $2,3 \%$ & $2,4 \%$ & $4,4 \%$ \\
\hline 2025 & $2,7 \%$ & $2,7 \%$ & $7,4 \%$ & 2048 & $2,5 \%$ & $2,6 \%$ & $5,6 \%$ \\
\hline 2026 & $2,4 \%$ & $2,4 \%$ & $5,9 \%$ & 2049 & $2,3 \%$ & $2,4 \%$ & $5,4 \%$ \\
\hline 2027 & $2,6 \%$ & $2,5 \%$ & $5,9 \%$ & 2050 & $2,3 \%$ & $2,4 \%$ & $5,4 \%$ \\
\hline
\end{tabular}

Fonte: Departamento econômico da empresa patrocinadora

Tabela 2 Ativos usados na Otimização

\begin{tabular}{c|c|c}
\hline $\begin{array}{c}\text { Indexador } \\
\text { pré fixado }\end{array}$ & $\begin{array}{c}\text { Nome do Ativo } \\
\text { LTN }\end{array}$ & $\begin{array}{c}\text { Número de Vencimento } \\
8\end{array}$ \\
\hline Selic & LFT & 75 \\
\hline IGP-M & NTN-C & 8 \\
\hline IPCA & NTN-B & 9 \\
\hline
\end{tabular}

\subsection{Definição dos Parâmetros}

Antes de apresentar os resultados, resta fazer uma breve descrição de como foram estabelecidos os parâmetros do modelo de otimização. Especificamente, serão apresentados como foram definidos os valores dos parâmetros $\sigma$ e $\lambda$ das equaçōes (4) e (5). Em primeiro lugar, cabe uma análise do que representam esses coeficientes. De forma simples, pode-se dizer 


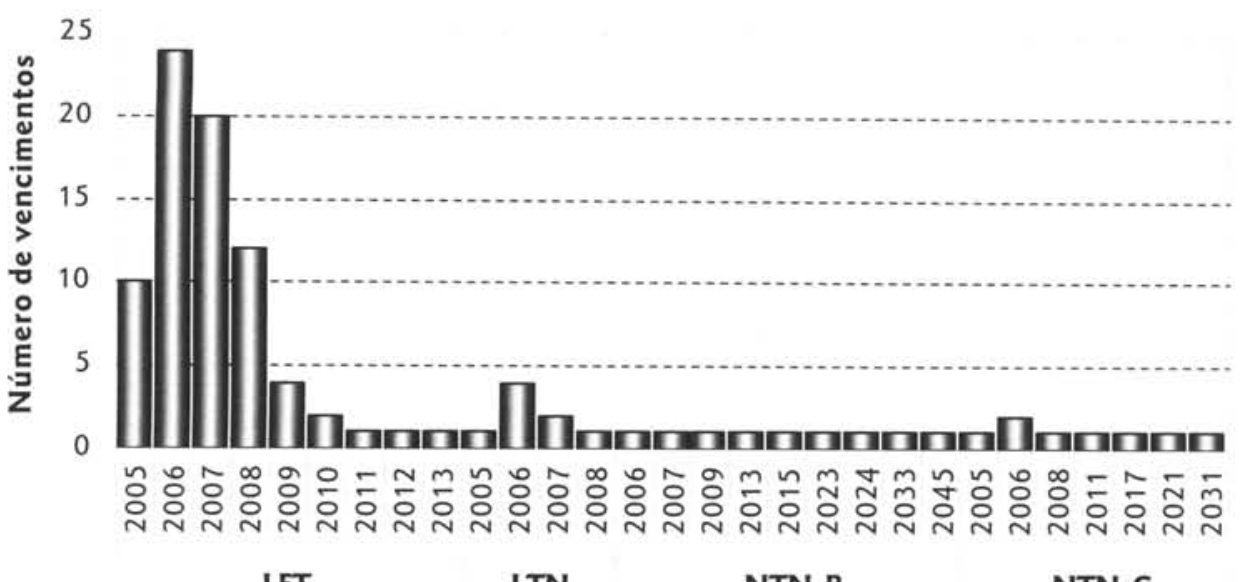

LFT LTN NTN-B NTN-C

Gráfico 2

Ativos usados na Otimização

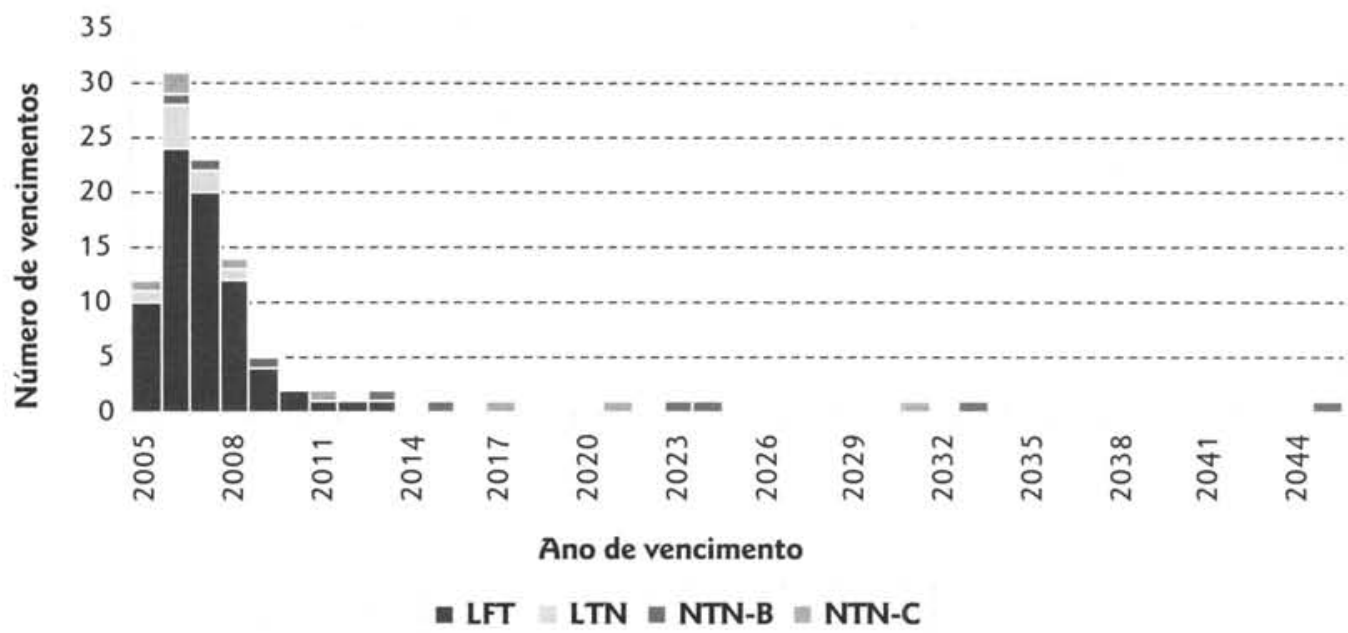

Gráfico 3

Ativos usados na Otimizaçāo

que o coeficiente $\lambda$ representa o grau absoluto de aversão ao risco, enquanto os desvios padrão, $\sigma$ de cada ativo, indicam o grau relativo dessa aversão. Isso ocorre porque, conforme indicam as equações (4) e (5), quando $\lambda$ aumenta, aumenta a penalização aplicada aos fluxos de caixa de todos os ativos. Porém, os ativos para os quais $\sigma$ é maior são relativamente mais afetados pelo aumento de $\lambda$. Além disso, vale lembrar que os coeficientes $\sigma$ representam, efetivamente, uma penalização à taxa de rentabilidade dos ativos expressa aproximadamente como porcentagem ao ano ${ }^{4}$.

Isso leva à proposta de definição dos coeficientes em duas etapas. Em primeiro lugar, definem-se os coeficientes $\sigma$ através da análise da taxa interna de retorno dos ativos. O Gráfico 40 apresenta as taxas internas de retorno de cada ativo calculadas com base nos fluxos de caixas esperados para eles.

Como era de se esperar, os ativos que apresentam as taxas de retorno mais atrativas são aqueles que carregam o maior risco para os participantes do mercado em geral, a saber, os ativos prefixados, que não apresentam proteção nem com relação às taxas de inflação, nem com relação às taxas de juros. Em seguida, em termos de rentabilidade, têm-se os ativos indexados à taxa Selic, seguido pelos ativos indexados às taxas de inflação.

É preciso definir, então, para o caso em análise, a gradação dos ativos em termos de risco. Para isso, faz-se necessário resgatar a informação de que o índice que corrige o passivo é o IGP-M. Dessa forma, é intuitivo que o ativo de menor risco 


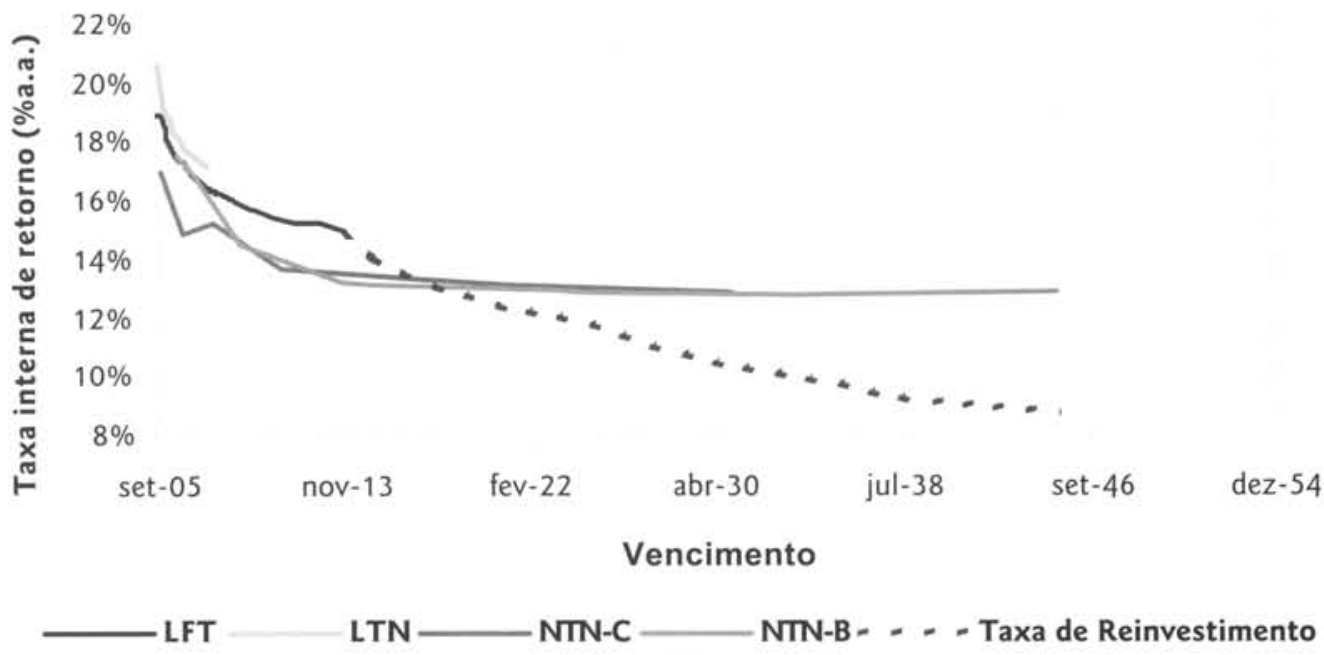

Gráfico 4

Taxas Internas de Retorno Projetadas

em relação ao passivo é a NTN-C, indexada ao IGP-M. Em seguida, seriam colocadas as NTN-Bs que, embora não sejam atreladas ao IGP-M, são atreladas a outro índice de inflação, o IPCA. Os títulos indexados à taxa Selic (LFTs) viriam a seguir. uma vez que apresentam mais proteção à inflação que os títulos prefixados (como o Banco Central do Brasil utiliza um regime de metas de inflação, é razoável admitir que maiores taxas de inflação levam a maiores taxas de juros, aumentando indiretamente a rentabilidade dos ativos indexados à taxa Selic). Os ativos prefixados seriam, portanto, os ativos de maior risco em relação ao passivo.

Assim. é possível escrever:

$$
\sigma_{N T N-C}<\sigma_{N T N-B}<\sigma_{L T F}<\sigma_{L T N}
$$

Foi fixada, arbitrariamente, uma correção de $0,5 \%$ a.a para os ativos indexados ao IGP-M, ou seja, toma-se $\sigma_{\text {NTN-c }}=$ $0.5 \%$. Isso quer dizer que o modelo exigirá uma "folga" financeira de cerca de $0,5 \%$ sobre um fluxo de caixa de 1 ano de uma NTN-C, assumindo-se um coeficiente de aversão ao risco $\lambda$ igual a 1. Grosso modo, o efeito dessa correçăo sobre o Gráfico 4 é o de deslocar a curva de rentabilidade das NTN-Cs em aproximadamente $0.5 \%$ para baixo. Como se pretende penalizar mais os outros ativos, em funçāo de seu risco maior, devem-se escolher valores para os demais $\sigma$ tais que as curvas de rentabilidade desses ativos sejam, adequadamente, ajustadas. São propostos, entāo, os valores de $\sigma_{\mathrm{NTN}-\mathrm{B}}=1,0 \% \sigma_{\mathrm{L} \pi \mathrm{T}}=$ $2.0 \%$ e $\sigma_{\text {LTN }}=2,5 \%$, que geram as estruturas de taxa de retorno apresentadas no Gráfico $5 \bullet$. Note-se que a correção para a taxa de reinvestimento é a mesma que para as LFTs, uma vez que a taxa overnight é diretamente atrelada à taxa Selic.

Pode-se notar que o efeito da escolha indicada foi de aproximadamente igualar as taxas de retorno dos ativos ao mesmo tempo em que (1) penaliza descasamentos em relação ao passivo e (2) mantém, em geral, a rentabilidade dos ativos indexados ao IGP-M superior à dos outros ativos.

Resta, agora, estabelecer o valor de $\lambda$. A variação desse coeficiente tem dois efeitos sobre os resultados. O primeiro é o de gerar uma carteira otimizada que privilegia ativos mais indexados ao passivo, ou seja, aqueles para os quais se definem os menores valores de $\sigma$. O segundo efeito é o de gerar um excesso de ativos em relação ao que seria necessário conforme o modelo sem aversão ao risco. Esses efeitos são positivos à medida que consistem exatamente nas respostas às limitações do modelo inicial apresentado. Porém, evidentemente, isso acarreta um custo que é o aumento do valor ótimo para função objetivo, ou seja, o aumento do valor necessário para montagem da carteira. Como esse valor necessário está limitado ao valor total de ativos do plano, pode-se usá-lo como uma informação conveniente para guiar a fixação do valor de $\lambda$. Para os valores viáveis da função objetivo, ou seja, inferiores ao patrimônio líquido do plano, pode-se, então, definir o valor de $\lambda$ através da preferência por risco do cliente, ou seja, apresentando-se o trade-off entre uma carteira mais aderente ao passivo e uma carteira com custo de montagem menor ${ }^{5}$. Nesse ponto, surge uma interessante idéia de que, através do modelo. pode-se calcular o superávit ou déficit econômico do plano baseado nos preços de mercado, que nada mais é do que o valor do patrimônio do plano subtraído do valor da função objetivo. Essa metodologia contrasta com a forma legal de se calcular o valor presente das obrigaçōes à taxa (arbitrária) de 6\% a.a. 


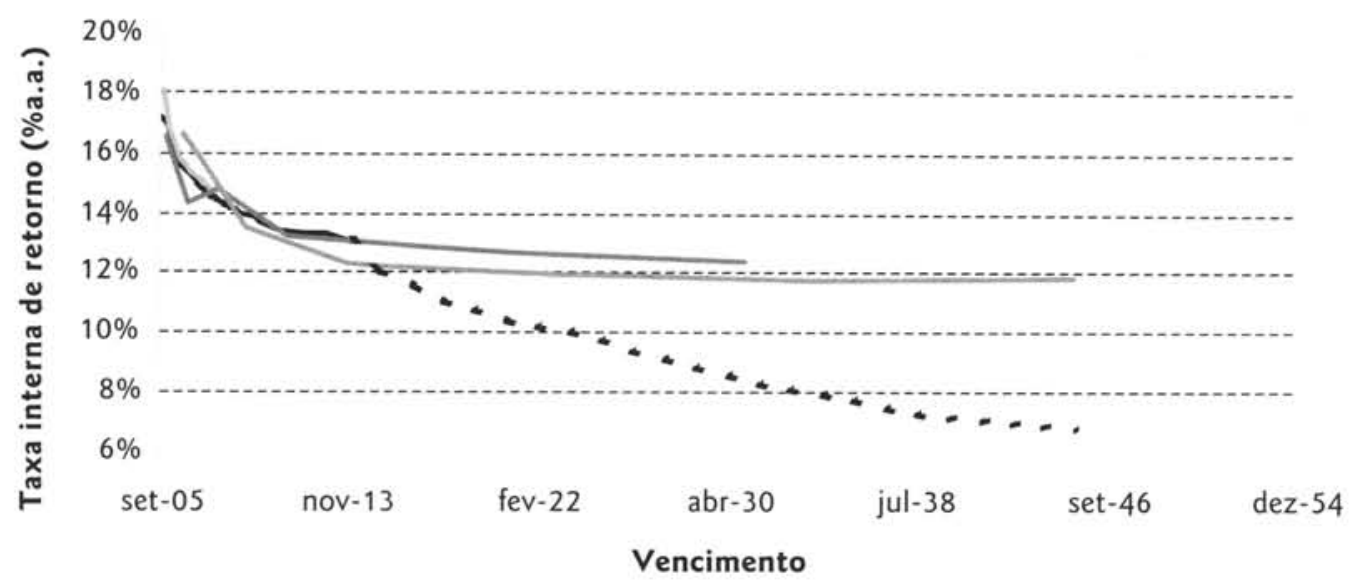

$\longrightarrow$ LFT LTN $\longrightarrow$ NTN-C NTN-B - - - Taxa de Reivestimento

Gráfico 5

Taxas de Retorno Aproximadas, ajustadas para $\lambda=1$

Dessa forma, o modelo foi implementado, utilizando-se 21 valores de $\lambda$ variando de 0,0 a 2,0 em intervalos de 0.1 . Os Gráficos 60 e 70 mostram a composiçāo percentual de cada carteira assim gerada em função de $\lambda$. em termos do tipo de ativo e do ano de vencimento. No Gráfico 6, é traçada, também. a curva do valor de montagem da carteira em função de $\lambda$. que ilustra o custo de se migrar para uma carteira que apresente menor risco em relação ao passivo.

De fato, à medida que o valor do coeficiente de aversão a risco aumenta, a exposição da carteira a ativos indexados à taxa de inflação, especialmente ao ICP-M. cresce consideravelmente. Ao mesmo tempo, o valor necessário para a montagem da carteira aumenta de $R \$ 69$ milhões $(\lambda=0)$ para $R \$ 81$ milhões $(\lambda=2)$. Outro efeito interessante que se observa é que ocorre uma concentração de prazos da carteira ao redor de 2013 (2008, 2011, 2007, 2021) à medida que se aumenta o valor do coeficiente de aversão ao risco. Isso ocorre porque, apesar de o modelo preferir o risco de reinvestimento ao risco de mercado, altos valores de $\lambda$ penalizam as taxas de reinvestimento e "forçam" o modelo a preferir vencimentos de ativos próximos aos vencimentos dos passivos.

Com base nesses gráficos e considerando-se o patrimônio líquido do plano na data da otimização (cerca de R\$ 85 miIhōes), decidiu-se utilizar um valor de $\lambda$ igual a 1,1, que representa a máxima proteção para o passivo dentro da viabilidade de ativos do plano, permitindo alguma folga para o pagamento de despesas da carteira. Observe que, caso a opção fosse por um valor menor de $\lambda$. surgiria um problema adicional de gestão, que o modelo não trata, de como investir o excedente.

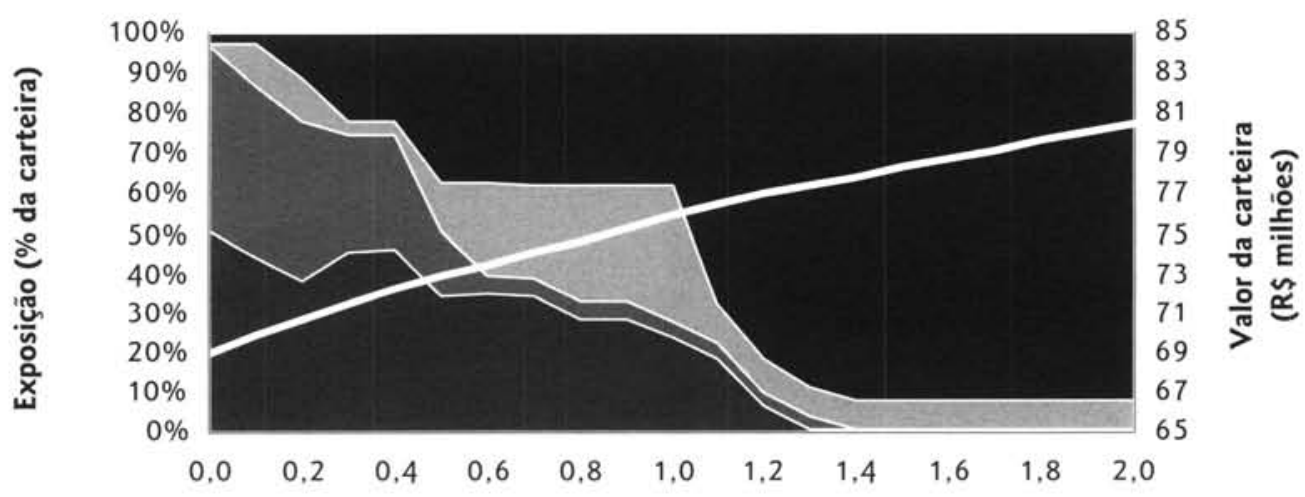

Aversão ao reisco

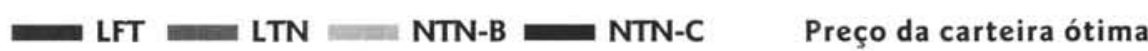

Gráfico 6

Carteiras Ótimas para Diferentes Valores de $\lambda$ (quebra por ativo) 


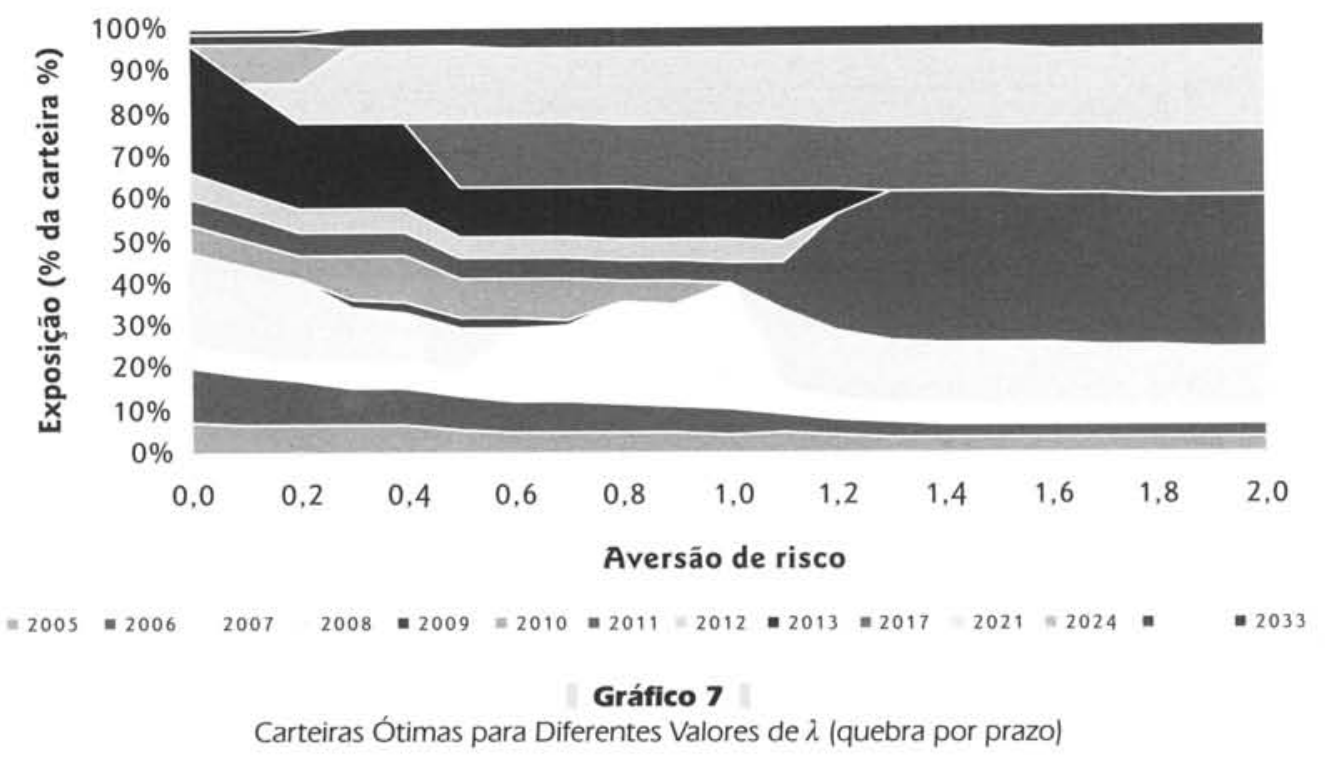

\section{ANÁLISE DOS RESULTADOS}

Tendo apresentado a metodologia de definição dos parâmetros e estabelecido os seus valores, segue a apresentação dos resultados obtidos. A título ilustrativo serão detalhados os resultados para duas situações, uma $\operatorname{com} \lambda=0$ e a outra com o valor escolhido para o parâmetro de aversão a risco $(\lambda=1,1)$. Os Gráficos $8 \mathbf{0}$ e 90 apresentam a composição das carteiras ótimas para esses dois casos. Os valores necessários para a montagem dessas carteiras são de, respectivamente, $R \$ 69$ milhōes e R\$ 77 milhōes.

Para ambos os casos, o valor do superávit inicial ótimo (caixa inicial) é zero. Os resultados mostram que, quando se utiliza $\lambda=0$, a carteira otimizada é formada, especialmente, pelos ativos de maior rentabilidade, ou seja, os ativos prefixados e os ativos indexados à taxa Selic (Gráfico 4). Para $\lambda=1,1$, a carteira privilegia aderência ao indexador do passivo e aderência aos prazos dos fluxos de caixa dos pagamentos. A maior aderência de prazos da carteira com aversão a risco pode ser observada nos Gráficos $10 \bullet$ e $11 \bullet$, nos quais foram traçados os gráficos dos fluxos de caixa nominais acumulados ano a ano. Observe-se que para a carteira com aversão ao risco, os valores acumulados caminham de forma muito mais próxima ao longo do tempo. Além disso, cabe destacar que, considerando-se as taxas de reinvestimento, há uma "folga" muito maior para o atendimento do passivo nessa carteira.

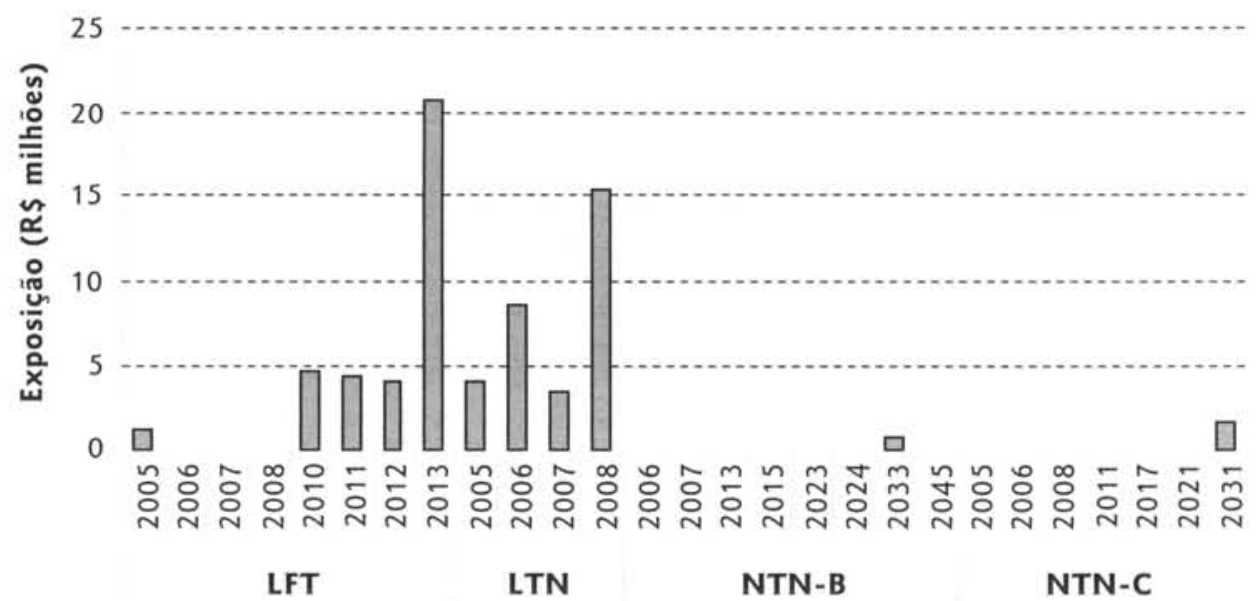

Gráfico 8

Composiçāo da Carteira Ótima para $\lambda=0$ 


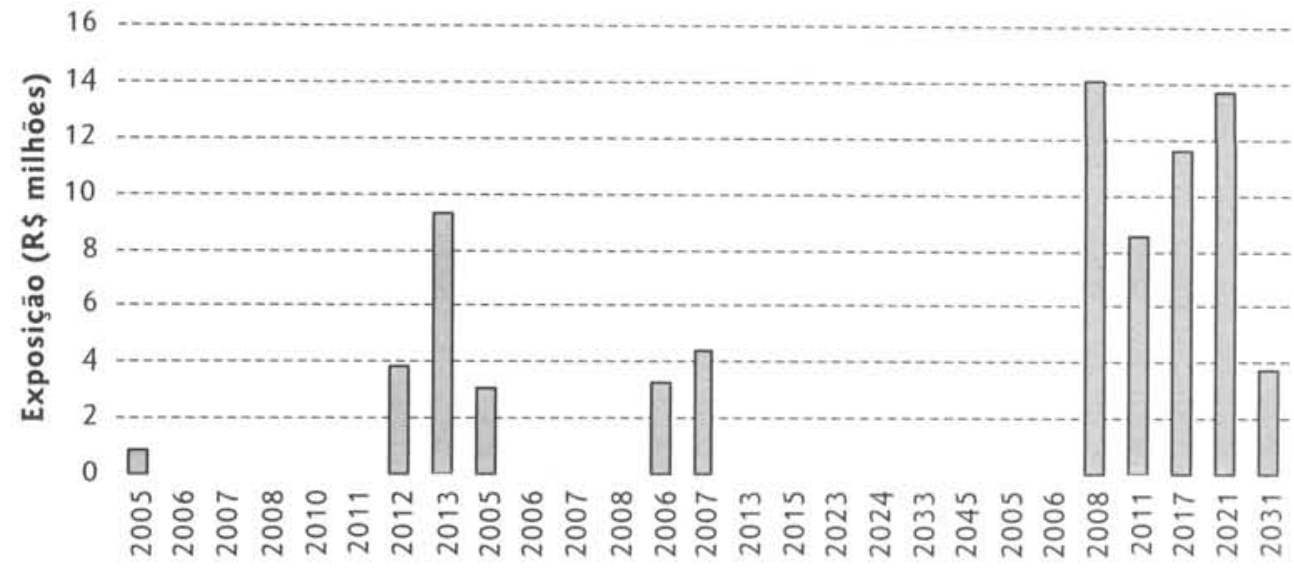

LFT

LTN

NTN-B

NTN-C

Gráfico 9

Composiçẫo da Carteira Ótima para $\lambda=1,1$

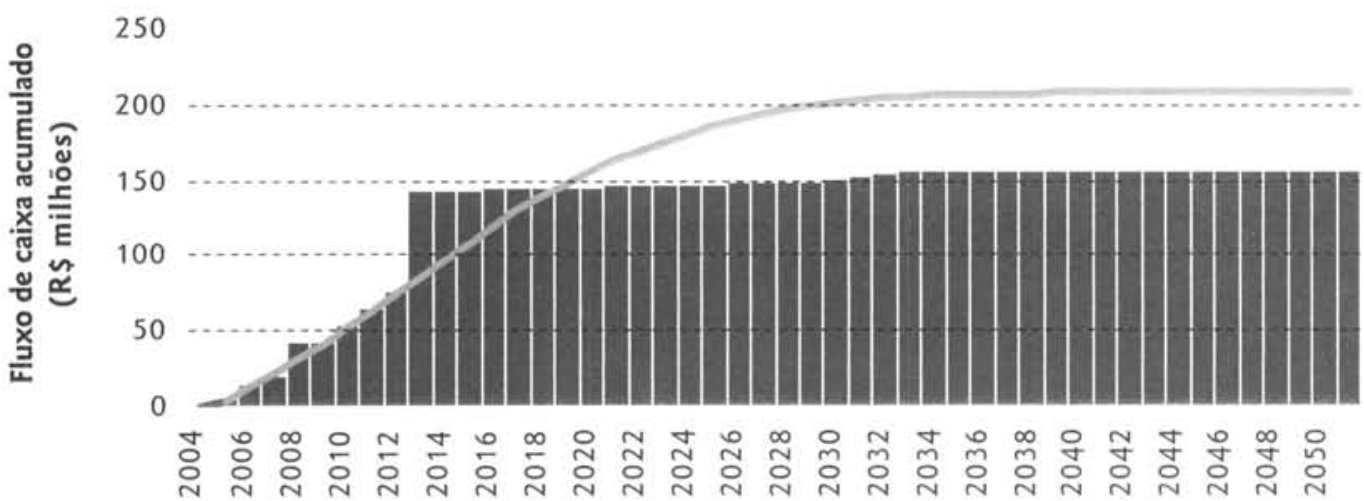

Data

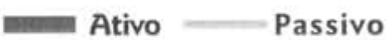

Gráfico 10

Fluxos de Caixa Acumulados para $\lambda=0$

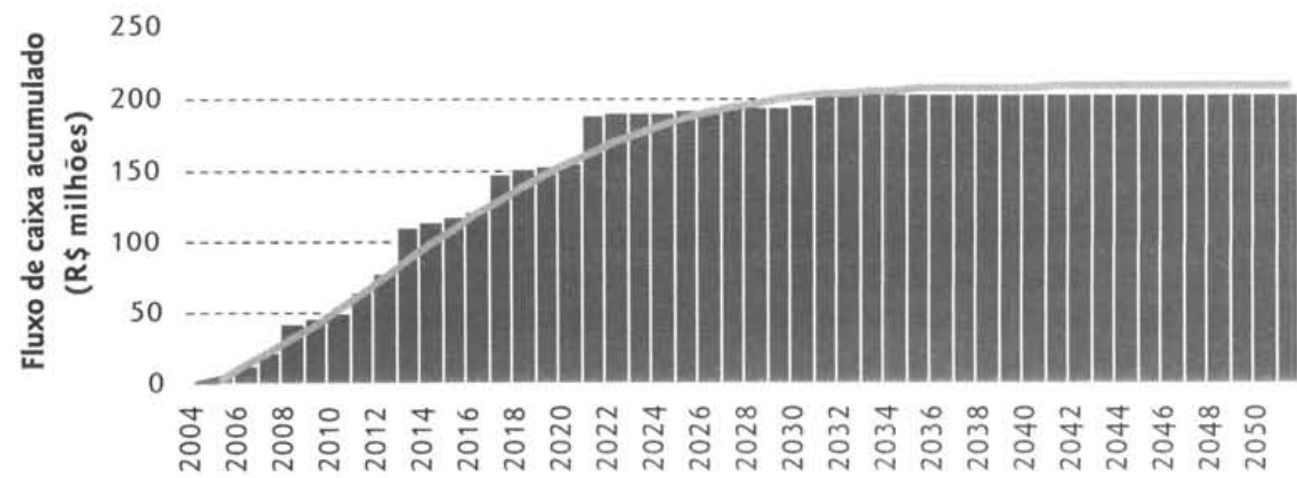

Data

Ativo $\longrightarrow$ Passivo

Gráfico 11

Fluxos de Caixa Acumulados para $\lambda=1,1$ 
Tabela 3 Características do Passivo e das Carteiras

\begin{tabular}{l|c|c|c}
\hline Valor da Carteira (R\$MM) & $\begin{array}{c}\text { passivo } \\
69,0\end{array}$ & $\begin{array}{c}\lambda=0 \\
69,0\end{array}$ & $\begin{array}{c}\lambda=1 \\
76,5\end{array}$ \\
\hline Taxa interna de retorno & $14,2 \%$ & $16,4 \%$ & $14,7 \%$ \\
\hline Duração & 3,8 & 2,9 & 4,0 \\
\hline Duração em percentual & $5,4 \%$ & $4,2 \%$ & $5,2 \%$ \\
\hline Duração de Macauley (anos) & 6,2 & 4,9 & 6,0 \\
\hline
\end{tabular}

Observe-se que o valor da carteira ótima, sem aversão a risco, pode ser interpretado como o valor presente do passivo às taxas de mercado, uma vez que constitui o montante necessário para se montar a carteira que, segundo as projeçōes da Tabela 1. atendem, exatamente, aos fluxos de caixa do passivo. Com isso, pode-se calcular uma taxa interna de retorno para o passivo, já que se tem seu "preço" e seus fluxos projetados (Gráfico 1). Adicionalmente, tendo a taxa interna de retorno, pode-se calcular a duração (duration) do passivo (FABOZZI, 2004) e compará-la com a duração das carteiras.

A Tabela 30 resume características selecionadas para as carteiras e para o passivo. A taxa interna de retorno para as carteiras foi calculada, por simplificação, através da média ponderada das taxas internas de retorno projetadas para cada ativo. A duração de cada carteira corresponde à variação financeira aproximada do seu valor para uma variação de um ponto porcentual da taxa interna de retorno. A duração em percentual corresponde à variação percentual aproximada do seu valor para uma variaçāo de um ponto porcentual da taxa interna de retorno. A duraçāo de Macauley corresponde à média ponderada do prazo de cada fluxo de caixa, na qual o peso utilizado é o valor presente desse fluxo (LUENBERGER, 1998). Os valores das durações são medidas da sensibilidade do preço das carteiras em relação às variaçōes das taxas de desconto, e, portanto, representam uma medida de risco das carteiras, que deve, no entanto, ser analisada relativamente ao passivo.

A análise da Tabela 3 leva a algumas conclusões interessantes. Com relação ao passivo, pode-se concluir que, baseado no cenário econômico traçado e nos ativos disponíveis, as obrigações podem ser atendidas com uma carteira de patrimônio igual a R\$69 milhões. Esse valor contrasta fortemente com o valor presente do passivo atuarial determinado conforme a legislação, que é de $R \$ 84.2$ milhōes $^{6}$.

Outra característica interessante das carteiras otimizadas é que, como era de se esperar, as suas duraçōes percentual e de Macauley são inferiores às do passivo. Isso decorre da imposição de que os fluxos de caixa dos ativos precedam os fluxos de caixa dos passivos.

\section{CONCLUSÃO}

O artigo apresentou um novo modelo para gestão de ativo/passivo (Asset/Liability Management), que possibilita a inclusão de ativos de renda fixa com fluxo incerto. Para o problema real analisado o modelo gerou uma carteira adequada para gestão dos ativos do fundo em questão, que leva em consideração não somente as expectativas de rentabilidade para os diversos ativos, mas também a composição do passivo e o risco de cada ativo em relação às obrigaçōes. Essa metodologia mostrou-se bastante superior à idéia de simplesmente se superar a meta atuarial de IGP-M mais $6 \%$ a.a. Além disso. a carteira gerada pôde ser, adequadamente, implementada tendo-se em vista as limitaçōes de recursos do plano, e indicou que a estimativa de superávit, calculado conforme a definição legal, é excessivamente conservadora.

Um cuidado importante na implementação do modelo refere-se à possível volatilidade da carteira quando ela é marcada a mercado. Isso pode se tornar um problema porque, por se tratar de uma carteira de títulos longos, pode haver grandes oscilaçōes de preço, que, por sua vez, podem acarretar flutuações excessivas do superávit atuarial. Portanto, para aplicação dos resultados do modelo, é crucial considerar-se a opção de se marcar os títulos longos "na curva de aquisição", ou seja, considerar que eles serão levados ao vencimento.

Com relação ao modelo apresentado neste artigo vale destacar que a maior contribuição é a de permitir um tratamento diferenciado para os diferentes riscos apresentados por cada ativo em relação ao passivo. Cabe observar, também, que essa metodologia é aplicável mesmo quando se aumenta o número de classes de ativos na otimização, e que, portanto, ela permite a inclusão, na otimização, de títulos privados, títulos indexados ao dólar, entre outros. O modelo não trata bem, no entanto, ativos que tenham valor residual no horizonte de análise, como, por exemplo, açōes ou títulos de renda fixa 
perpétuos. Por fim, o modelo pode ser aperfeiçoado tanto na direção do refinamento das equações de correção do fluxo de caixa como também no sentido de que, na forma apresentada, trata-se de um modelo estático.

\section{Referências Bibliográficas}

BOENDER G. C. E.. A hybrid simulation/optimisation scenario model for asset/liability management. European Journal of Operational Research. V. 99, n. 1, p. 126-135, 1997.

BOULIER J.; HUANG S.; TAILLARD, G.. Optimal management under stochastic interest rates: the case of a protected defined contribution pension fund. Insurance: Mathematics and Economics, v. 28, n. 2, p. 173-189, 2001.

CAIRNS, A. J. G.; PARKER G.. Stochastic pension fund modeling. Insurance: Mathematics and Economics, v. 21, n. 1, p. 43-79, 1997.

DAHL, H.; MEERAUS, A.; ZENIOS, S. A.. Some financial optimization models: I Risk Management. IN ZENIOS, S. A. (org.). Financial optimization. Cambridge: Cambridge University, 1993.

Some financial optimization models: II Financial Engineering. IN ZENIOS, S. A. (org.). Financial optimization. Cambridge: Cambridge University, 1993.

FABOZZI, F.J.. Fixed Income Analysis for the Chartered Financial Analyst@ Program. New Hope, Frank J. Fabozzi Associates, 2004.

FRANGOS, C.; ZENIOS, S.A.; YAVIN, Y.. Computation of feasible portfolio control strategies for an insurance company using a discrete time asset/liability model. Mathematical and Computer Modelling. V. 40, n. 3-4, p. 423-446, 2004.

JÚDICE; J.J.; RIBEIRO, C. O.; SANTOS, P.J.. Análise Comparativa dos Modelos de Selecção de Carteiras de Acçōes de Markowitz e Konno. Investigação Operacional, v. 23, p.211-224, 2003.

KONNO, H.; YAMAZAKI, H.. Mean-absolute deviation portfolio optimization model and its application to Tokio stock market. Management science, v. 37, p. 519-531, 1991.

LUENBERGER, D. G.. Investment Science. Nova York: Oxford University Press, 1998.

MARKOWITZ, H. M.. Portfolio Selection. Journal of Finance, v. 7, n. 1, p. 77-91, 1952.

PAPI, M.; SBARAGLIA, S.. Optimal asset-liability management with constraints: A dynamic programming approach. Applied Mathematics and Computation, In Press, 2005.

PFLUG, G.. Scenario tree generation for multiperiod financial optimization by optimal discretization, Mathematical Programming, B., v. 89 , n. 2, p. $251-257,2001$.

SAAD, N.; RIBEIRO, C.O.. Modelos determinísticos de gestāo de ativo/passivo: uma aplicação no Brasil. Revista de Contabilidade e Finanças, n. 34 , p. 50-62, 2004.

STEINBACK, M.C.. Markowitz revisited: mean variance models in financial portfolio analysis. SIAM review, v. 43, p. 31-85, 2001.

\section{NOTA - Endereço dos autores}

HSBC Investments

Av. Brig. Faria Lima, 3064

Sã̃o Paulo-SP

01451-000
Universidade de São Paulo

Escola Politécnica

Av. Almeida Prado, trav.2, 128

São Paulo - SP

05508-900 